\title{
Sympathetic Nerve Fibers in Human Cervical and Thoracic Vagus Nerves
}

\author{
Atsuko Seki, $\mathrm{MD}^{\dagger}$, Hunter R. Green ${ }^{\dagger}$, Thomas D. Lee, MD, $\mathrm{PhD}^{\dagger}$, LongSheng Hong ${ }^{\dagger}$, Jian \\ Tan, MS ${ }^{\ddagger}$, Harry V. Vinters, MD $^{\dagger}$, Peng-Sheng Chen, MD, FHRS ${ }^{\ddagger}$, and Michael C. Fishbein, \\ $\mathrm{MD}^{\dagger}$ \\ †Department of Pathology and Laboratory Medicine, David Geffen School of Medicine at UCLA, \\ Los Angeles, CA
}

¥Krannert Institute of Cardiology, Division of Cardiology, Department of Medicine, Indiana University School of Medicine, Indianapolis, IN

\begin{abstract}
Background-Vagus nerve stimulation therapy (VNS) has been used for chronic heart failure (CHF), and is believed to improve imbalance of autonomic control by increasing parasympathetic activity. Although it is known that there is neural communication between the VN and the cervical sympathetic trunk, there are few data regarding the quantity and/or distribution of the sympathetic components within the VN.
\end{abstract}

Objective-To examine the sympathetic component within human VN and correlate these with the presence of cardiac and neurologic diseases.

Methods-We performed immunohistochemistry on 31 human cervical and thoracic VNs (total $104 \mathrm{VNs}$ ) from autopsies and we reviewed the patients' records. We correlated the quantity of sympathetic nerve fibers within the VNs with cardiovascular and neurologic disease states.

Results-All 104 VNs contain TH positive (sympathetic) nerve fibers; the mean TH positive areas were $5.47 \%$ in right cervical, $3.97 \%$ in left cervical, $5.11 \%$ in right thoracic, and $4.20 \%$ in left thoracic VN. The distribution of TH positive nerve fibers varied from case to case: central, peripheral, or scattered throughout nerve bundles. No statistically significant differences in nerve morphology were seen between diseases in which VNS is considered effective (depression and $\mathrm{CHF}$ ), and other cardiovascular diseases, or neurodegenerative disease.

Conclusion-Human VNs contain sympathetic nerve fibers. The sympathetic component within the VN could play a role in physiologic effects reported with VNS. The recognition of

(C) 2014 The Heart Rhythm Society. Published by Elsevier Inc. All rights reserved.

Address for Correspondence and Reprint Request: Atsuko Seki, M.D., Department of Pathology and Laboratory Medicine, UCLAcenterfor the Health Sciences., 10833 Le Conte Ave, Los Angeles, CA 90095, USA, Business telephone: 310 794-7498, Fax: 310 794-4161, ASeki@mednet.ucla.edu.

Publisher's Disclaimer: This is a PDF file of an unedited manuscript that has been accepted for publication. As a service to our customers we are providing this early version of the manuscript. The manuscript will undergo copyediting, typesetting, and review of the resulting proof before it is published in its final citable form. Please note that during the production process errors may be discovered which could affect the content, and all legal disclaimers that apply to the journal pertain.

Conflicts of Interest: None of the authors have conflicts of interest to disclose 
sympathetic nerve fibers in the VNs may lead to better understanding of the therapeutic mechanisms of VNS.

\section{Keywords}

Cervical vagus nerves; Sympathetic nerves; Ganglion cells; Heart failure; Vagal nerve stimulation

\section{INTRODUCTION}

Cardiovascular abnormalities are associated with an imbalance in the cardiac autonomic nervous system, ${ }^{1-4}$ typically, characterized by increased sympathetic activity and decreased parasympathetic (vagal) activity. ${ }^{2-6}$ Chronic vagal stimulation therapy (VNS) is believed to improve the imbalance of cardiac autonomic control by increasing parasympathetic activity. Accordingly, vagus nerve stimulation therapy has been introduced as a novel therapy for chronic heart failure (CHF), ${ }^{1,4-9}$ and has demonstrated improvement in symptoms. ${ }^{4,6,9}$

The anatomy of the vagus nerve is variable between species. ${ }^{10,11}$ In human, the vagus nerve is largely anatomically separated from sympathetic nerves. ${ }^{10,12}$ However, there is communication between the vagus nerve and the cervical sympathetic trunk. ${ }^{10,12}$ Onkka et al. performed immunohistochemical studies in dogs, and reported the percentage and the distribution of sympathetic nerve fibers within the cervical vagus nerve. ${ }^{13}$ Kawagishi et al. using immunohistochemistry showed that catecholaminergic fibers were present in the human vagus nerve. ${ }^{12}$ Sympathetic components within the vagus nerve may contribute to both therapeutic and adverse events associated with VNS therapy. However, there are limited data regarding the location and quantity of sympathetic nerve component within human vagus nerve. There is no information on how any sympathetic component in the vagus nerve might correlate with clinical disease. We examined the vagus nerves from 31 patients at autopsy, described the sympathetic component, and correlated the structural components of the vagus nerves with the presence of major cardiac and neurologic diseases.

\section{METHODS}

\section{Histological study for vagus nerves}

At autopsy, we harvested left and right cervical and thoracic vagus nerves from 20 men and 11 women (Table 1). In all cases consent for research was included with the consent for autopsy. Cervical vagus nerves were identified deep to the jugular vein and common carotid artery. Then, the dissection proceeded into the more distal parts of the vagus nerves that entered the thoracic cavities. The right and left cervical vagus nerves were sampled from the region where vagus nerve stimulation (VNS) therapy is applied. The right and left thoracic vagus nerves were sampled $5 \mathrm{~cm}$ inferior to the aortic arch. Nerves were fixed in $10 \%$ formalin for one hour, then transferred to $70 \%$ ethyl alcohol. Tissues were processed routinely in graded alcohols, cleared in xylol, and paraffin embedded. Paraffin blocks were cut into 5- $\mu \mathrm{m}$ thick sections. Samples were cut to obtain both cross and longitudinal sections. Sections were stained with hematoxylin and eosin. Immunohistochemical staining was also performed on paraffin-embedded cross sections. We used tyrosine hydroxylase (TH) antibodies (Mouse Monoclonal Anti-Tyrosine Hydroxylase SIGMA, T1299) to 
identify adrenergic nerve fibers and sympathetic ganglion cells. Anti-Choline Acetyltransferase (ChAT) antibodies (Rabbit polyclonal to Choline Acetyltransferase, abcam, ab68779) were used to identify parasympathetic ganglion cells. Glass slides stained with TH were scanned with a digital slide scanner at 20x (Aperio XT scanner, Aperio Technologies, Vista, CA). Digital image analysis software (Definiens' Tissue Studio, Definiens Inc., Parsippany, NJ) was used to calculate the cross sectional area of the nerve and TH positive areas within the vagus nerves. The TH positive area was divided by the cross sectional area. Figure 1 shows the methods that were used to quantify the TH positive area.

\section{Sympathetic nerve fibers and clinical disease}

In order to determine if there was any correlation of the presence of sympathetic nerve fiber with clinical disease, we reviewed the records from the 31 patients. Demographic data included gender, age, ethnicity, and history of cardiovascular and neurological diseases.

\section{Statistics}

The groups were evaluated for statistically significant differences using the non-parametric exact Wilcoxon Mann-Whitney rank sum test for two groups and the Kruskal-Wallis rank sum test for more than two groups. Non-parametric correlations were performed using the Spearman's rank correlation test. A total of 21 tests for statistical significance were performed. The resulting $\mathrm{p}$-values were adjusted for multiple testing using a Bonferroni correction factor of 21 .

\section{RESULTS}

The median age for these 31 cases was 64 years (34-99 years). Twenty patients (64.5\%) were Caucasians, four (12.9\%) Hispanic, one (3.2\%) Asian, one (3.2\%) Native American, and five (16.1\%) of unknown ethnicity. Disease status in these patients is shown in Table 1. Data regarding histologic finding are shown in Table 2. No statistically significant differences were identified between the four vagus nerve groups for bundle number (uncorrected $\mathrm{p}$-value $=0.125$, corrected $\mathrm{p}$-value $=1$ ) or cross sectional area (uncorrected $\mathrm{p}$ value $=0.008$, corrected $\mathrm{p}$-value $=0.178$ )

TH positive nerve fiber areas within the vagus nerves varied from patient to patient. However, no statistically significant difference was seen in TH positive area fraction between the four vagus nerve groups (uncorrected $\mathrm{p}$-value $=0.661$, corrected $\mathrm{p}$-value $=1$ ). TH positive nerve fibers could be central, peripheral, or scattered throughout nerve bundles (Figure 2). About one third of the cross sections consisting of 3 RCVN, 11 RTVN, 6 LCVN, and 12 LTVN had strongly TH positive nerve fiber bundles in the periphery of the vagus nerves. Surprisingly, ten vagus nerves contained 12 ganglion cells. Of nine ganglion cells, in serial sections seven were positive for ChAT, and two were positive for $\mathrm{TH}$.

For each case, the available bundle numbers, cross sectional areas, and TH positive area fractions were averaged over the four vagus nerve groups (Table 2). For the 31 cases, the mean bundle number average was 8.83 , the mean cross sectional area average was 1.54 $\mathrm{mm}^{2}$, and the mean $\mathrm{TH}$ positive area fraction average was $4.94 \%$. No statistically significant 
differences were seen based on sex or ethnicity (Table 3). No statistically significant correlations were seen between age and bundle number average (correlation coefficient $=$ 0.280 , uncorrected $\mathrm{p}$-value $=0.127$, corrected $\mathrm{p}$-value $=1$ ), age and cross sectional area average (correlation coefficient $=-0.219$, uncorrected $\mathrm{p}$-value $=0.236$, corrected $\mathrm{p}$-value $=$ 1 ), or age and TH positive area fraction average (correlation coefficient $=0.186$, uncorrected $\mathrm{p}$-value $=0.315$, corrected $\mathrm{p}$-value $=1)$.

\section{Clinical correlation}

Table 4 lists the major clinical/autopsy diagnoses and TH positive areas. There was no statistically significant association with the presence of structural cardiovascular or neurologic diseases, or whether or not the patient suffered from CHF or depression, or both.

\section{DISCUSSION}

The autonomic nervous system plays an important role in maintaining normal cardiac rhythm and rate. ${ }^{4,7}$ The balance of sympathetic and parasympathetic tone regulates cardiac autonomic control. ${ }^{1,4}$ Cardiovascular abnormalities, such as hypertension, heart failure, and coronary artery disease are associated with an imbalanced cardiac autonomic nervous system. ${ }^{1-3,14}$ The imbalance is generally characterized by increased sympathetic activity and decreased parasympathetic activity. ${ }^{2-6,14}$ Reduced vagal activity has been related to increased mortality, life-threatening arrhythmia, and sudden cardiac death in late stages of heart failure. 1, 2, 5, 6, 9, 15 Physiologically, parasympathetic stimulation affects preload and results in slowing of the heart rate and decreased cardiac output. ${ }^{2}$ Therefore, it has been thought that modulation of vagal activity had potential for the treatment of cardiovascular disease.

Indeed, VNS therapy has recently been introduced as a novel therapy for heart failure. ${ }^{1,4-9}$ The basic idea of VNS for CHF is to modify the autonomic imbalance by stimulating the vagus nerve. Animal studies showed that VNS had an antifibrillatory effect, prevented ventricular fibrillation induced by acute $\mathrm{MI}$ in the setting of a healed MI, and improved symptoms of heart failure. ${ }^{2}$ A pilot VNS study for CHF was performed in 8 patients. ${ }^{9}$ This study was followed by a multi-center, open-label phase II study of right cervical VNS in 32 patients with CHF. ${ }^{6}$ In both studies, significant improvements of New York Heart Association heart failure score, quality of life, and 6 minute walk test were observed. One hypothesis was that VNS resulted in slowing of heart rate and thereby improving cardiac function. However, heart rate reduction was seen only in $37.5 \%$ in the former study. ${ }^{9}$ In the latter study, the acute change of heart rate was modest, and the average heart rate change was not significant. ${ }^{6}$ Therefore, these authors concluded that the change in heart rate was not the main effect of VNS therapy. ${ }^{6}$ Since heart rate reduction was only moderate, other mechanisms for cardioprotection have been suggested. These include anti-apoptotic and anti-inflammatory effects of VNS. ${ }^{16}$

Chronic VNS therapy has been used as an FDA-approved therapy for drug-refractory epilepsy and depression. $5,7,8,15,17$ Generally adverse cardiac events, such as episodes of bradycardia are not observed during VNS therapy in the patients with epilepsy. The left vagus nerve is the one stimulated in VNS therapy for epilepsy. The left vagosympathetic 
trunk innervates the atrioventricular node in the dog. Stimulation of the left vagosympathetic trunk does not cause changes in heart rate. ${ }^{18}$ There are, however, several reports of cardiac effects during cervical VNS for epilepsy. ${ }^{19},{ }^{20}$ No patient with cardiovascular disease was included in these studies. No clinically relevant cardiac effects were observed. ${ }^{19,20}$ Interestingly, two of five patients had the occurrence of tachycardia of uncertain cause. ${ }^{20}$

The anatomy of the vagus nerve has been shown to be variable among species. ${ }^{10,11}$ In human, the vagus nerve is anatomically separated from the sympathetic trunk. ${ }^{10,12}$ In contrast, the vagus nerves are intermingled and interconnected with sympathetic fibers in some animals. This complex is called the vagosympathetic trunk. ${ }^{10,11}$ Randall et al. reported the presence of adrenergic nerve fibers within the cervical vagosympathetic trunk in dogs. These adrenergic fibers directly distributed to the heart. ${ }^{21}$ In one canine study, the cardiac responses caused by the stimulation of the cervical vagosympathetic trunk depended on the content of the nerve fibers. ${ }^{22}$ In human, a neural communication between the vagus nerve and cervical sympathetic trunk sometimes exists. ${ }^{10,12}$ However the significance of sympathetic component is not well understood. Recently, Onkka et al. performed a quantitative study of sympathetic nerve fibers within the cervical vagus nerve in normal dogs. ${ }^{13}$ The sympathetic nerve fibers occupied $0.03-2.80 \%$ of the cervical vagus nerve cross-sectional area. ${ }^{13}$ Randall et al. have shown that the adrenergic nerve fibers within the cervical vagosympathetic trunk directly distributed to the heart in dogs. ${ }^{21}$ Kawagishi et al. studied the vagus nerves and their branches, including superior and recurrent laryngeal branches, the superior and inferior cardiac branches, and the pulmonary branches, in 12 human cadavers. ${ }^{12}$ These authors reported that catecholaminergic fibers were present in both cervical and thoracic vagus trunks and all their branches. ${ }^{12}$ However, no quantitative examination or clinicopathological correlation were reported.

Our study indicates that the vagus nerve consistently has a component of sympathetic nerve fibers in right/left and cervical/thoracic trunk. The distributions of sympathetic nerve fibers were variable, and occupied $0.00-21.63 \%$ of total cross sectional area. Since the vagus nerve stimulator is usually placed around the left cervical vagus nerve in the carotid sheath for epilepsy, and around the right cervical vagus nerve for CHF, we compared the amount of $\mathrm{TH}$ fibers in left and right vagus nerves. There was no significant difference in $\mathrm{TH}$ positive nerve fibers between right and left vagus nerves, nor between cervical and thoracic vagus nerves. Although both central and peripheral autonomic nervous systems are affected by many diseases, ${ }^{23}$ there was no significant relationship between the percentage of sympathetic nerve fibers within the vagus nerve and the diseases we examined in this study.

VNS therapy may activate not only parasympathetic nerve fibers but also these sympathetic nerve fibers. In our study, one third of subjects had discrete bundles of sympathetic nerve fibers at the periphery of their vagus nerves. Onkka et al. reported that $\mathrm{TH}$ positive nerves were typically present at the periphery of nerve bundles in normal dogs. ${ }^{13}$ In our human study, while the vagus nerve had bundles of sympathetic nerve fibers at the periphery, there were also more diffusely placed sympathetic nerve fibers throughout the cross section of the vagus nerve. Kawagishi et al. reported the inferior cervical ganglion had TH positive fibers in the periphery and suggested that the inferior ganglion would be the possible vagal site receiving the sympathetic fibers. ${ }^{12}$ Although the significance of the peripheral localization is 
not clear in our study, the peripheral localization also may influence sympathetic effects during vagus nerve stimulation therapy.

The anatomical and physiological bases, and mechanism of VNS are not clear, and thought to be complex. ${ }^{24}$ For clinical use of vagal stimulation, the frequencies range from 20 to 30 Hz. ${ }^{25}$ Different responses are seen in EEG recordings by stimulation of different fibers of the vagus. ${ }^{25}$ The vagus nerve has about $80 \%$ afferent sensory fibers and $20 \%$ efferent fibers. ${ }^{17,24,25}$ Efferent fibers include a-motorneurons and parasympathetic nerve fibers. Afferent fibers are sensory fibers, and there are A-fibers, B-fibers, and C-fibers. ${ }^{24,} 25$

A-fibers carry afferent visceral information and motor input, B-fibers carry parasympathetic input, and C-fibers carry afferent visceral information. ${ }^{25}$ Morphologically, A-fibers and B fibers are myelinated, and C-fibers are non-myelinated. ${ }^{25}$ It was shown that the antiepileptic potency of VNS was related to the C-fibers of vagus nerve in rats. ${ }^{26}$ But, another study in rats demonstrated the destruction of C-fibers had no effect on seizure suppression. ${ }^{27} \mathrm{In}$ human, stimulation is administrated to the afferent fibers of the vagus nerve, thus, the stimulation influences both cerebral hemispheres. ${ }^{24}$ The effective stimulation in human VNS for epilepsy is thought to be primarily mediated by afferent vagal A- and B-fibers. ${ }^{24}$ It has been reported that in studies of physiological responses to VNS in human, efferent Afibers were activated and no afferents fibers were excited. ${ }^{28}$ Stimulation of afferent vagus nerve fibers is preferred for the treatment of epilepsy because the origin of epilepsy is considered to be cortical. Efferent stimulation is thought to cause side effects. ${ }^{24}$ However, which nerve fibers are involved or responsible for the effect of VNS, efferent or afferent, A-, $\mathrm{B}-$, or C- fibers, is not clear. Onkka reported that in dogs the TH positive nerve fibers in vagus nerves were nonmyelinated. ${ }^{13}$ It has also been reported that the unmyelinated C-fibers were involved in VNS applied in rats. ${ }^{26}$ In spite of a number of investigations, which fibers are responsible for the benefit and adverse events for VNS remains unknown. ${ }^{24,26-28}$

Our study has limitations associated with any autopsy study. While our study of human vagus nerves is the largest to date, we only studied 31 autopsied hospitalized patients. Most of our patients were elderly, and had cardiovascular and neurodegenerative diseases, and other comorbid conditions. Autonomic abnormalities and neuropathy increases with aging. ${ }^{29}$ We did not have young normal control patients. Both central and peripheral autonomic nervous systems are affected by many diseases, in addition to the ones we identified. ${ }^{23}$ The vagus nerve innervates many different systems and organs, including the cardiovascular system. The cardiac branches and the intrinsic cardiac nervous system are known to be important; ${ }^{30}$ however, we did not study the intrinsic cardiac nervous system, nor the vagus cardiac branches. Further investigations of larger numbers of patients including younger and more normal subjects and the cardiac branches and intrinsic cardiac nervous system may be more revealing in demonstrating associations between vagus nerve components and disease.

\section{CONCLUSION}

In conclusion, human vagus nerves consistently contain sympathetic nerve fibers. The distribution and degree of sympathetic nerve fibers within the vagus nerve are variable 
between cases. The greater presence of sympathetic nerve fibers within the vagus nerve in some patients could play a role in some of the effects reported with VNS.

\section{Acknowledgments}

The authors gratefully acknowledge the technical assistance of Dr. Clara Magyar, as well as Audley Broadey, and Keith Ellis for support regarding the autopsies.

Financial support:

This study was supported in part by NIH Grants P01 HL78931, R0171140, a Medtronic-Zipes Endowment and the Indiana University Health-Indiana University School of Medicine Strategic Research Initiative (PSC), and an endowment from the Piansky Family Trust (MCF).

\section{ABBREVIATIONS}

$\begin{array}{ll}\text { ChAT } & \text { cholineacetyltransferase } \\ \text { CHF } & \text { chronic heart failure } \\ \text { TH } & \text { tyrosine hydroxylase } \\ \text { VN } & \text { cervical vagus nerve } \\ \text { VNS } & \text { vagal nerve stimulation }\end{array}$

\section{REFERENCES}

1. Kobayashi M, Massiello A, Karimov JH, Van Wagoner DR, Fukamachi K. Cardiac autonomic nerve stimulation in the treatment of heart failure. Ann Thorac Surg. 2013 Jul.96:339-345. [PubMed: 23747176]

2. Schwartz PJ. Vagal stimulation for heart diseases: from animals to men. - An example of translational cardiology. Circ J. 2011; 75:20-27. [PubMed: 21127379]

3. Hauptman PJ, Schwartz PJ, Gold MR, Borggrefe M, Van Veldhuisen DJ, Starling RC, Mann DL. Rationale and study design of the increase of vagal tone in heart failure study: INOVATE-HF. Am Heart J. 2012 Jun.163:954.e951-962.e951. [PubMed: 22709747]

4. Schwartz PJ, De Ferrari GM. Vagal stimulation for heart failure: background and first in-man study. Heart Rhythm. 2009 Nov.6:S76-S81. [PubMed: 19880077]

5. Schwartz PJ. Vagal stimulation for the treatment of heart failure: a translational success story. Heart. 2012 Dec.98:1687-1689. [PubMed: 22962285]

6. De Ferrari GM, Crijns HJ, Borggrefe M, et al. Chronic vagus nerve stimulation: a new and promising therapeutic approach for chronic heart failure. Eur Heart J. 2011 Apr.32:847-855. [PubMed: 21030409]

7. Zhang Y, Mazgalev TN. Arrhythmias and vagus nerve stimulation. Heart Fail Rev. 2011 Mar. 16:147-161. [PubMed: 20559719]

8. Anholt TA, Ayal S, Goldberg JA. Recruitment and blocking properties of the CardioFit stimulation lead. Journal of neural engineering. 2011 Jun.8:034004. [PubMed: 21543838]

9. Schwartz PJ, De Ferrari GM, Sanzo A, Landolina M, Rordorf R, Raineri C, Campana C, Revera M, Ajmone-Marsan N, Tavazzi L, Odero A. Long term vagal stimulation in patients with advanced heart failure: first experience in man. European journal of heart failure. 2008 Sep.10:884-891. [PubMed: 18760668]

10. Randall, WC.; Armour, JA., editors. Neural Regulation of the Heart. 1977. Oxford University Press Inc; 1977. Chapter 2: Gross and Microscopic Anatomy of the Cardiac Intervention; p. 14-30. 
11. Armour, JA.; Hopkins, DA. Nervous Control of Cardiovascular Function. Oxford University Press Inc; 1984. Chapter 2: Anatomy of the Extrinsic Efferent Autonomic Nerves and Ganglia Innervating the Mammalian Heart; p. 20-45.

12. Kawagishi K, Fukushima N, Yokouchi K, Sumitomo N, Kakegawa A, Moriizumi T. Tyrosine hydroxylase-immunoreactive fibers in the human vagus nerve. Journal of clinical neuroscience : official journal of the Neurosurgical Society of Australasia. 2008; 15:1023-1026. [PubMed: 18617399]

13. Onkka P, Maskoun W, Rhee KS, Hellyer J, Patel J, Tan J, Chen LS, Vinters HV, Fishbein MC, Chen PS. Sympathetic nerve fibers and ganglia in canine cervical vagus nerves: localization and quantitation. Heart Rhythm. 2013 Apr.10:585-591. [PubMed: 23246597]

14. Zamotrinsky AV, Kondratiev B, de Jong JW. Vagal neurostimulation in patients with coronary artery disease. Auton Neurosci. 2001 Apr 12.88:109-116. [PubMed: 11474540]

15. Cleland JG, Coletta AP, Clark AL, Cullington D. Clinical trials update from the American College of Cardiology 2009: ADMIRE-HF, PRIMA, STICH, REVERSE, IRIS, partial ventricular support, FIX-HF-5, vagal stimulation, REVIVAL-3, pre-RELAX-AHF, ACTIVE-A, HF-ACTION, JUPITER, AURORA, and OMEGA. European journal of heart failure. 2009 Jun.11:622-630. [PubMed: 19468023]

16. De Ferrari GM, Schwartz PJ. Vagus nerve stimulation: from pre-clinical to clinical application: challenges and future directions. Heart Fail Rev. 2011 Mar.16:195-203. [PubMed: 21165697]

17. Park MC, Goldman MA, Carpenter LL, Price LH, Friehs GM. Vagus nerve stimulation for depression: rationale, anatomical and physiological basis of efficacy and future prospects. Acta neurochirurgica Supplement. 2007; 97:407-416. [PubMed: 17691329]

18. Randall WC, Rohse WG. The augmentor action of the sympathetic cardiac nerves. Circ Res. 1956 Jul.4:470-475. [PubMed: 13330193]

19. Galli R, Limbruno U, Pizzanelli C, Giorgi FS, Lutzemberger L, Strata G, Pataleo L, Mariani M, Iudice A, Murri L. Analysis of RR variability in drug-resistant epilepsy patients chronically treated with vagus nerve stimulation. Auton Neurosci. 2003 Aug 29.107:52-59. [PubMed: 12927227]

20. Frei MG, Osorio I. Left vagus nerve stimulation with the neurocybernetic prosthesis has complex effects on heart rate and on its variability in humans. Epilepsia. 2001 Aug.42:1007-1016. [PubMed: 11554886]

21. Randall WC, Priola DV, Pace JB. Responses of individucal cardiac chambers to stimulation of the cervical vagosympathetic trunk in atropinized dogs. Circ Res. 1967 May.20:534-544. [PubMed: 6057686]

22. Randall WC, Armour JA. Regional vagosympathetic control of the heart. The American journal of physiology. 1974 Aug.227:444-452. [PubMed: 4854069]

23. Hans, J.; ten Donkelaar, VN.; Lammens, Martin; Sebastiaan Overeem, aAK. Clinical Neuroanatomy. Heidelberg: Springer-Verlag Berlin; 2011.

24. Vonck K, Boon P, Van Roost D. Anatomical and physiological basis and mechanism of action of neurostimulation for epilepsy. Acta neurochirurgica Supplement. 2007; 97:321-328. [PubMed: 17691318]

25. Ruffoli R, Giorgi FS, Pizzanelli C, Murri L, Paparelli A, Fornai F. The chemical neuroanatomy of vagus nerve stimulation. Journal of chemical neuroanatomy. 2011 Dec.42:288-296. [PubMed: 21167932]

26. Woodbury DM, Woodbury JW. Effects of vagal stimulation on experimentally induced seizures in rats. Epilepsia. 1990; 31:S7-S19. [PubMed: 2226368]

27. Krahl SE, Senanayake SS, Handforth A. Destruction of Peripheral C-Fibers Does Not Alter Subsequent Vagus Nerve Stimulation-Induced Seizure Suppression in Rats. Epilepsia. 2001; 42:586-589. [PubMed: 11380564]

28. Binks AP, Paydarfar D, Schachter SC, Guz A, Banzett RB. High strength stimulation of the vagus nerve in awake humans: a lack of cardiorespiratory effects. Respiration physiology. 2001 Sep. 127:125-133. [PubMed: 11504585]

29. Kiba T. The role of the autonomic nervous system in liver regeneration and apoptosis--recent developments. Digestion. 2002; 66:79-88. [PubMed: 12428066] 
30. Armour, Ja; Murphy, Da; Yuan, BX.; Macdonald, S.; Hopkins, Da. Gross and microscopic anatomy of the human intrinsic cardiac nervous system. The Anatomical record. 1997; 247:289298. [PubMed: 9026008] 


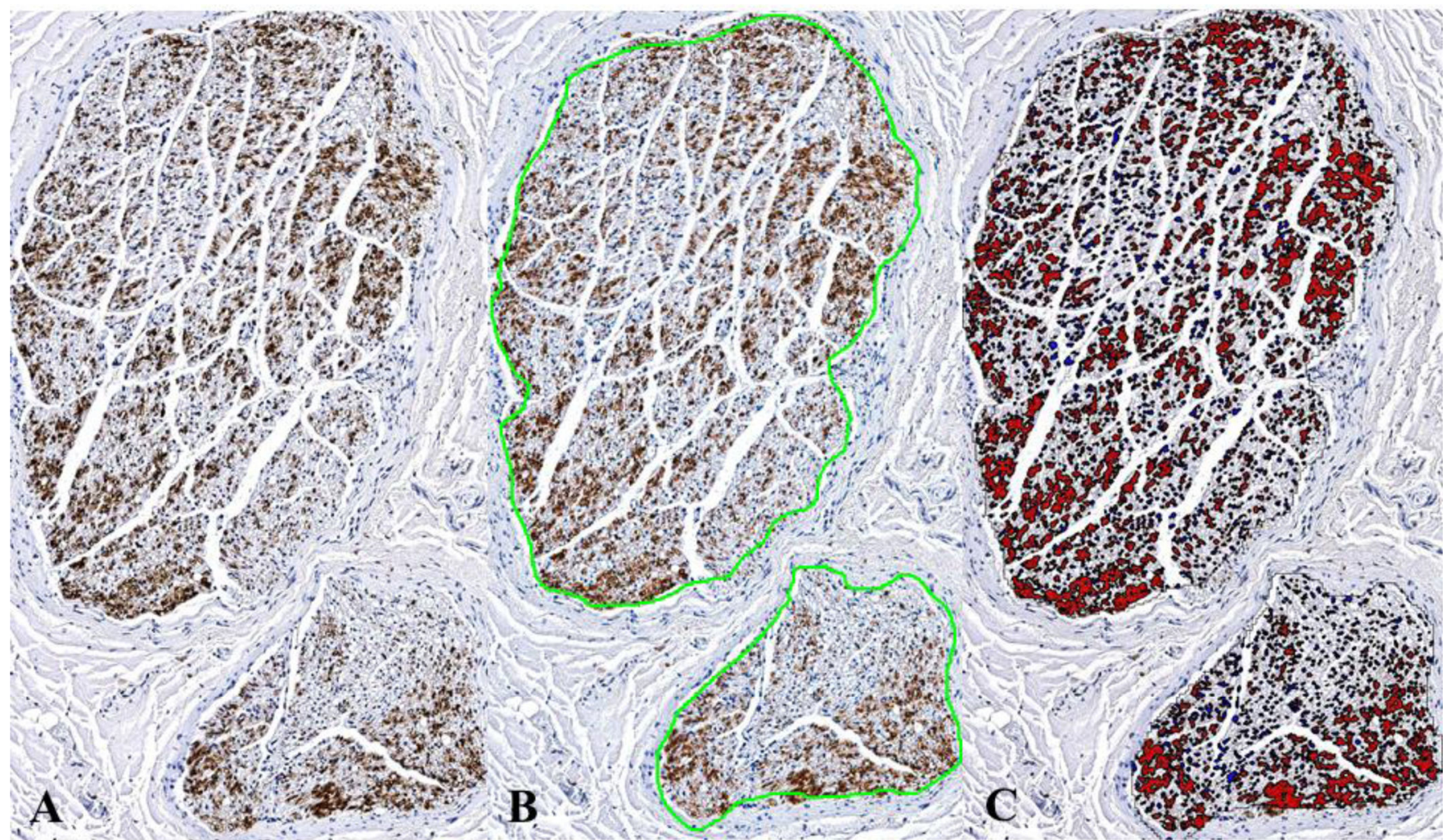



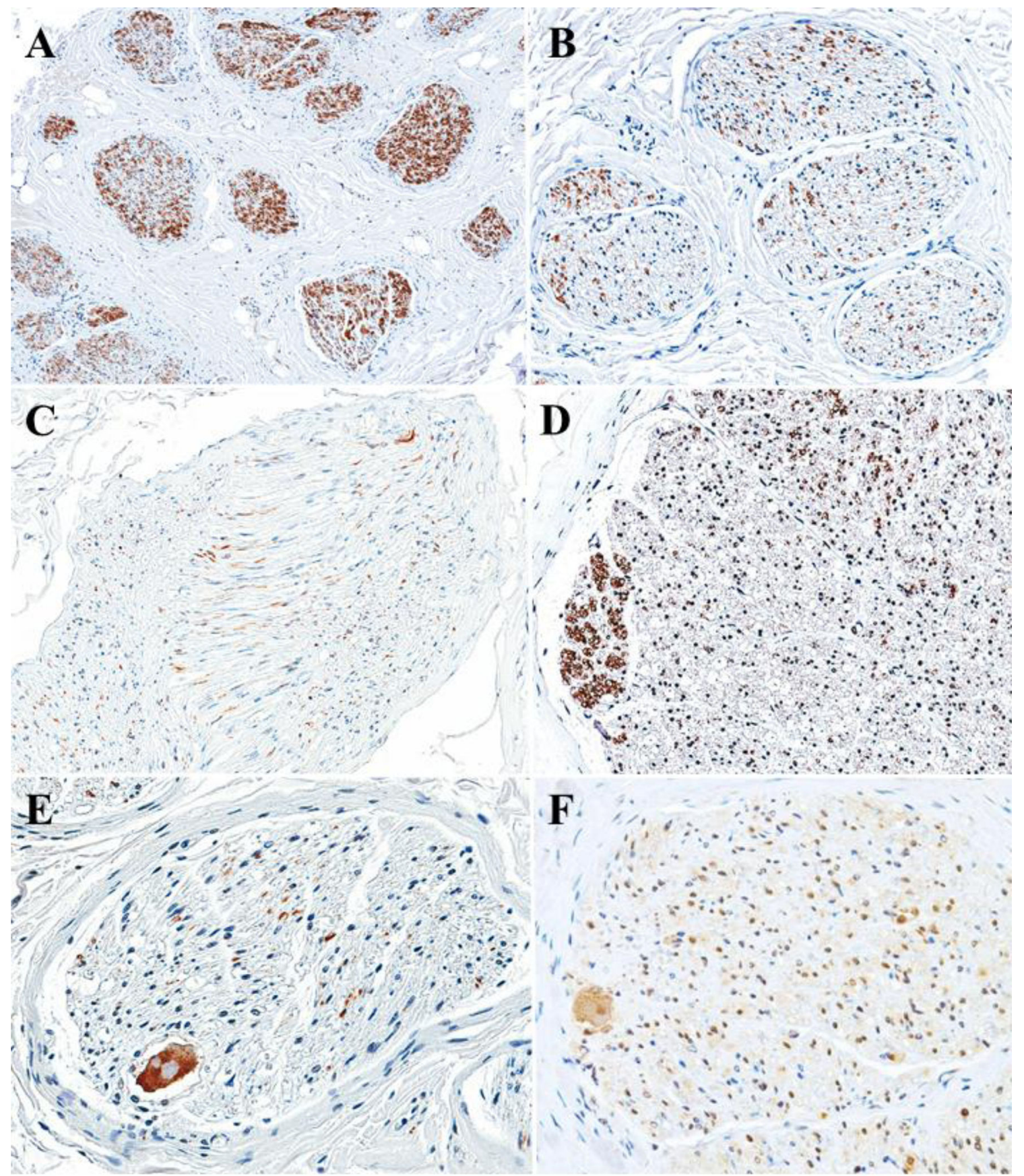


\section{Table 1}

\section{Disease states}

Total Number of Cases

Cardiovascular Diseases

Neurodegenerative Disease

Diseases Related to Vagus Nerve Stimulation

Therapy: Chronic heart failure and/or Depression

\section{1}

20 cases $(64.5 \%)$ including 12 diagnoses of hypertension, 10 coronary artery disease or myocardial infarction, 5 atrial fibrillation, 3 sudden cardiac death, and 2 ventricular tachycardia

12 cases $(38.7 \%)$ including 11 diagnoses of Alzheimer's disease, 2 Parkinson disease, 1 Huntington disease, and 1 multiple system atrophy

10 cases $(32.3 \%)$ including 4 diagnoses of chronic heart failure and 7 depression 


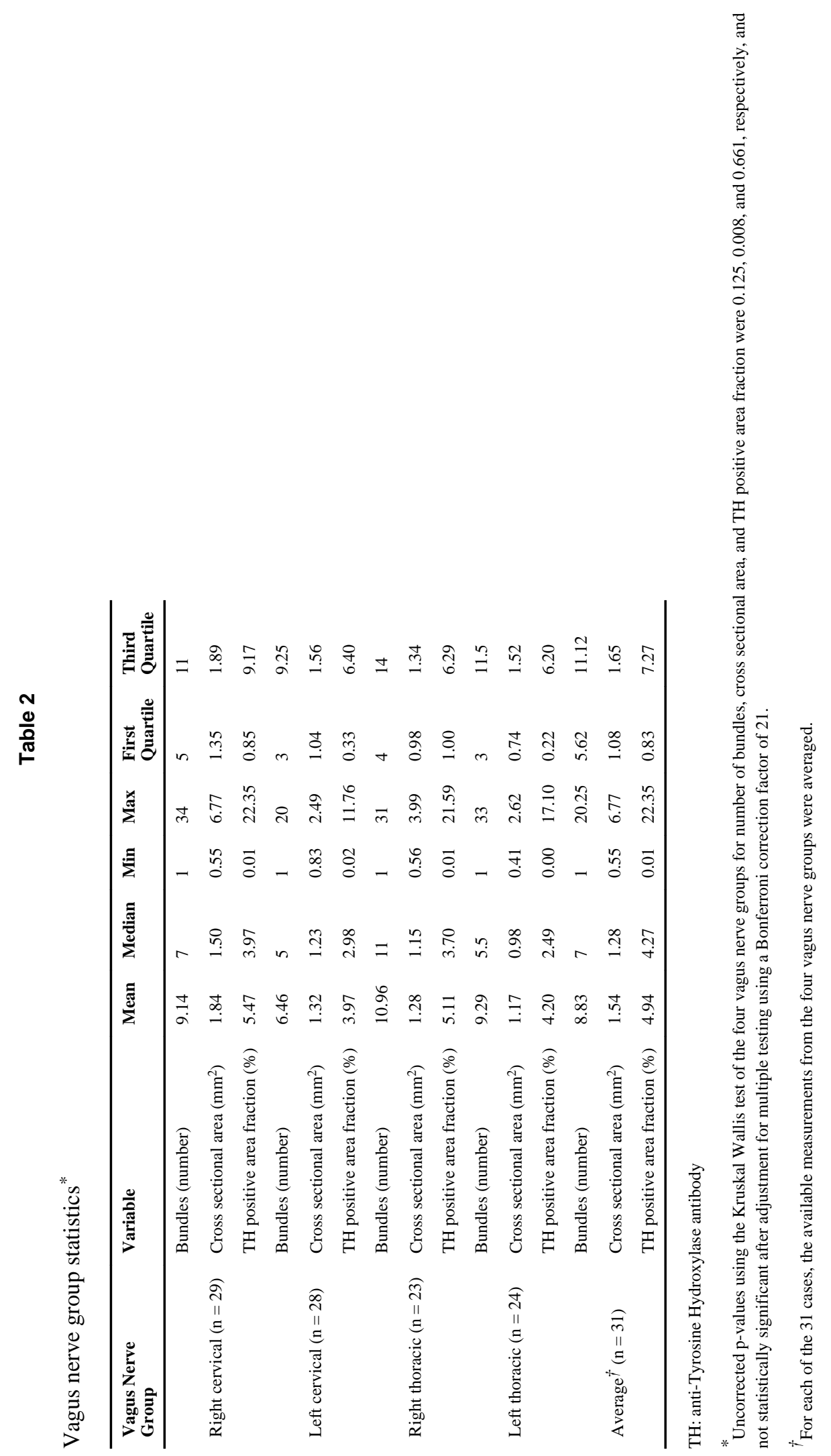




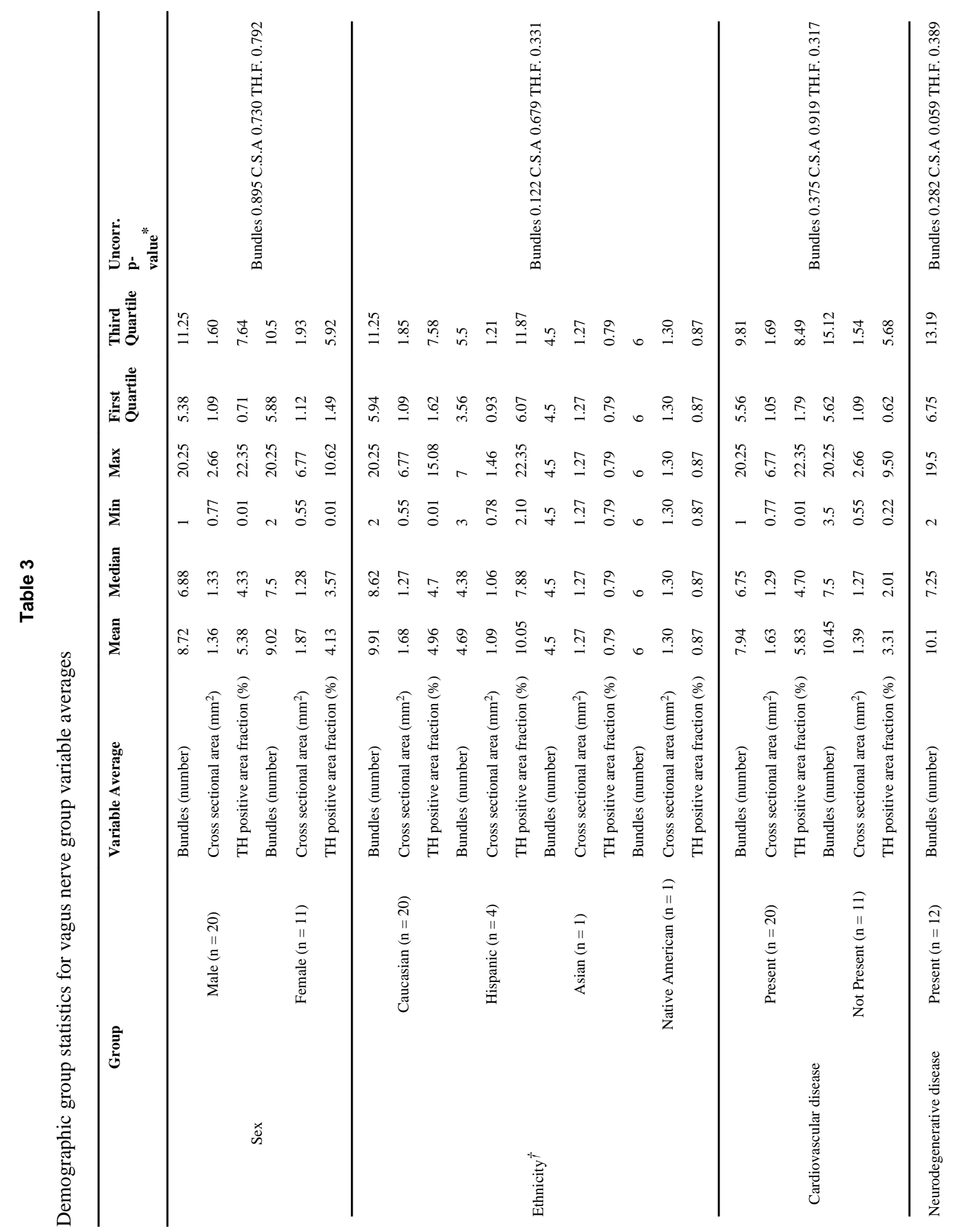

Heart Rhythm. Author manuscript; available in PMC 2015 August 01. 


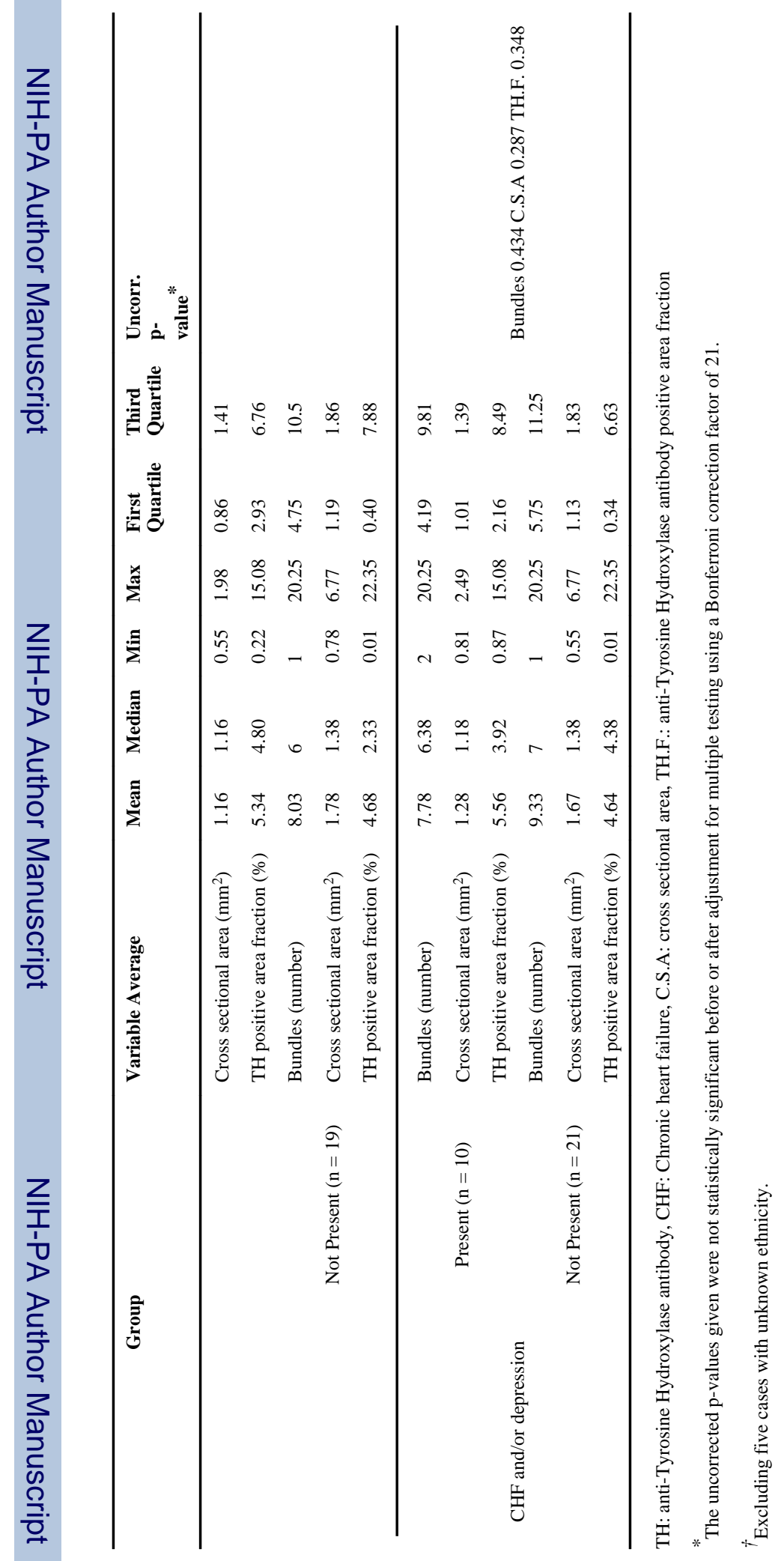


